\title{
MULTI-OBJECTIVE DESIGN OPTIMIZATION OF THE LOW-YIELD STEEL SHEAR PANEL DAMPER
}

\author{
Y. Liu ${ }^{1}$, M. Shimoda ${ }^{2}$ \\ ${ }^{1}$ Department of Advanced Science and Technology, Toyota Technological Institute \\ (liuyang@toyota-ti.ac.jp) \\ ${ }^{2}$ Department of Advanced Science and Technology, Toyota Technological Institute
}

\begin{abstract}
This paper presents a shape optimization methodology for the design of the lowyield steel shear panel damper without stiffeners. The optimization was carried out as a multiobjective optimization problem to maximize the total absorbing energy and minimize the max. accumulated equivalent plastic strain of the shear panel damper under cyclic loading. With application of the optimization methodology of the response surface methodology combined with the design of experiment technique, firstly, finite element analysis with isotropic/kinematic hardening model was used to simulate the cyclic elasto-plastic behavior instead of experimental approach, and reliability of the numerical solutions was confirmed by comparing to previous experimental results. Then, based on the numerical analysis, the shape parameters effects and their interactions were investigated and second order polynomials were fitted to obtain the regression equations of the total absorbing energy and the max. accumulated equivalent plastic strain. Finally, the regression equations were applied to constitute the multi-objective functional by using the weighed sum method, and maximization problem of the formulated multi-objective functional was solved.
\end{abstract}

Keywords: Shear panel damper, Multi-objective optimization, Energy absorption, Accumulated plastic stain, Response surface methodology, Design of experiment.

\section{INTRODUCTION}

Shear panel damper (SPD) made of low yield steel has high level of passive energy dissipation capacity as a consequence of inelastic deformation of the low yield steel, and has been received considerable interest in the last two decades. When installed into building and bridge structures, it is expected to partially divert the input seismic energy into the SPDs and effectively reduce the seismic responses of the structures under strong earthquake loads. To be a type of hysteretic damper, properly design of the SPD (stiffened or unstiffened) is strongly required to sustain high deformation capacity and repetition durability for low cycle fatigue under cyclic seismic loading, especially for the application in bridge structures, which demand large range of shear deformation. If the SPD is designed unreasonably, the clacks 
should initiate at edges or corners of the shear panel in the early stage of cyclic loading due to the stress concentration, and grow along with cycles, that will decrease the energy absorption capacity drastically. In focusing on improving the deformation capacity and repetition durability, recently, some experimental and analytical researches have been carried out by varying the panel shape or installing the stiffeners on the left and right sides of the SPD for the application in bridge structures [1][2][3]. However, most of researches are confined to be empirical methods or analytical researches dealing with direct problems, the shape optimization of obtaining the maximization of deformation capacity and total absorbing energy has not been studied.

The studies on optimization of elastic and elasto-plastic structures have been extensively investigated during the past 30 years, and a number of useful algorithms and methodologies are developed(e.g. [4][5]). As a practical and effective optimum design methodology for nonlinear problem, the response surface methodology (RSM) combined with the design of experiment (DOE) technique is currently applied to nonlinear design optimization problems, such as optimization problems of crushing energy absorbing of the automobile body and boxtype column structures [6]. In this paper, a multi-objective design optimization of the SPD was studied by the response surface approximation methodology and the technique of designof-experiment. Since deformation capacity of the SPD can be evaluated by the max. accumulated equivalent plastic strain at a cyclic shear deformation, the objective functions are the max. accumulated equivalent plastic strain and the total absorbing energy. Instead of experimental approach, the cyclic behavior of SPD subjected to cyclic loading is studied by sophisticated finite element method (FEM) with isotropic/kinematic hardening model, and a comparison between numerical simulation and experimental result was made and precision of the numerical simulation was confirmed. Then an orthogonal array is employed to arrange the design point using the technique of design of experiment, and numerical simulations of the SPDs whit various shape parameters were carried out. Based on the numerical results, the influences of the shape parameters to responses of the max. accumulated equivalent plastic strain and the energy absorbing behaviors are investigated to obtain the regression equations of the two objective functions. Finally, the response surface methodology was adopted to solve the multi-objective optimization problem, and to obtain the optimal shape parameters of the SPD.

\section{2 NUMERICAL ANALYSIS}

\subsection{Analysis model}

The initial shape of SPD, which is a $156 \times 156 \mathrm{~mm}$ square plate with uniform plate thickness of $\mathrm{tw}=12 \mathrm{~mm}$, is shown in Figure 1. The upper and lower edges of the panel are groove welded to plates. Cyclic lateral load was applied at the upper plate, and the loading history is shown in Figure 2, in which the increments of the shear displacement in each loading cycle are $\pm 1 \delta \mathrm{y}$, where $\delta \mathrm{y}=5 \mathrm{~mm}$ is the shear yield displacement corresponding to the $0.2 \%$ offset yield stress of the material. 


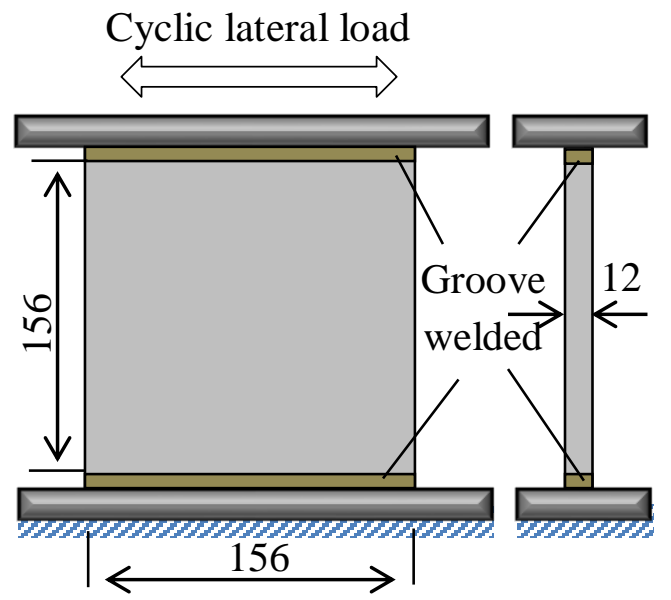

Figure 1. Initial SPD

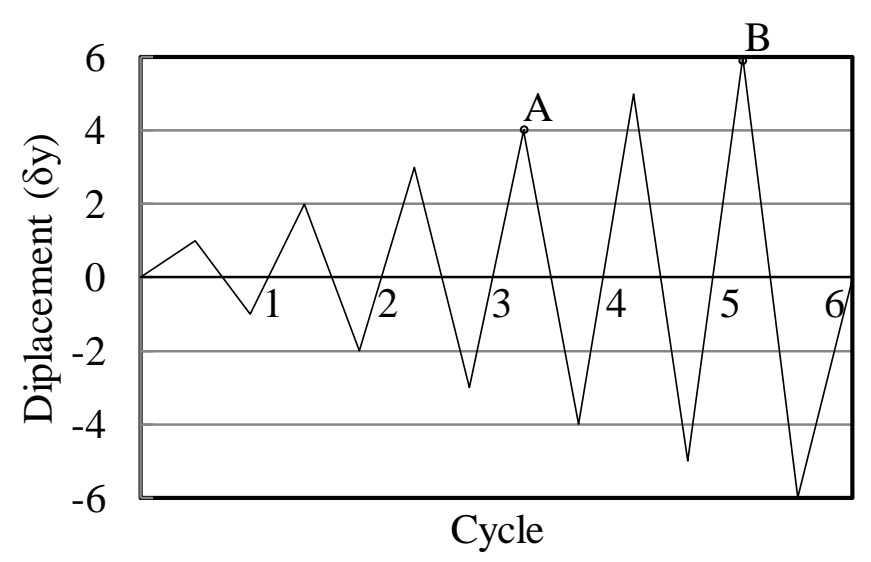

Figure 2. Loading history

\subsection{Material properties and constitutive law}

The material properties of low-yield 100(LY100) steel were measured by tensile coupon test and the obtained stress-strain curves are shown in Figure 3. The yield strength defined $0.2 \%$ offset value of LY100 is $80.1 \mathrm{~N} / \mathrm{mm}^{2}$ and elongation reaches $60 \%$, which is about three times of SS400 mild steel.

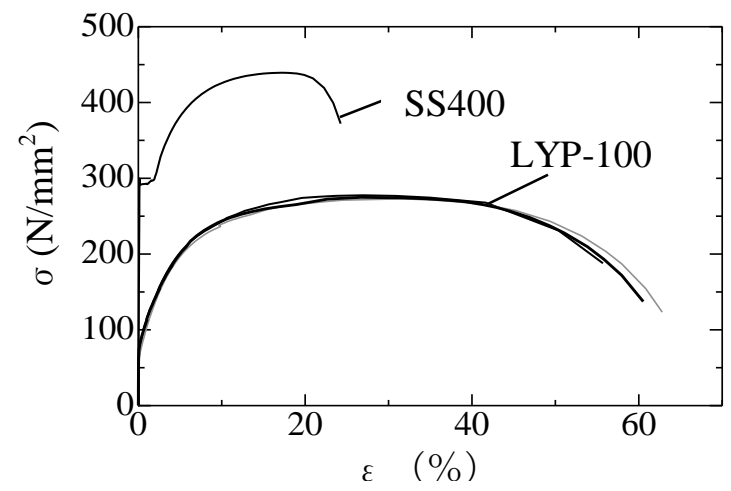

Figure 3. Stress-strain curves in tension

The cyclic elasto-plastic behavior of SPD subjected to cyclic loading is simulated by ABAQUS with a combined isotropic/kinematic hardening model [7], which was employed as constitutive law to describe the material cyclic behavior accurately. The combined hardening model consists of two components: a nonlinear kinematic hardening component and an isotropic hardening component.

The kinematic hardening component, that describes the translation of the yield surface in stress space through the back-stress $\alpha$ is defined to be an additive combination of a purely kinematic term (linear Ziegler hardening law) and a relaxation term (the recall term), which introduces the nonlinearity.

$$
\dot{\alpha}=C(\sigma-\alpha) \dot{\varepsilon}^{p l} / \sigma_{0}-\gamma \alpha \dot{\varepsilon}^{p l}
$$


where $C$ are the initial kinematic hardening moduli, and $\gamma$ determine the rate at which the kinematic hardening moduli decrease with increasing plastic deformation. The kinematic hardening law can be separated into a deviatoric part and a hydrostatic part; only the deviatoric part has an effect on the material behavior.

The isotropic hardening behavior of the model defines the evolution of the yield surface size $\sigma^{0}$, as a function of the equivalent plastic strain $\bar{\varepsilon}^{p}$. This evolution can be introduced by the simple exponential law:

$$
\sigma^{0}=\sigma_{0}+Q_{\infty}\left(1-e^{-b \bar{\varepsilon}^{p}}\right)
$$

where $\sigma_{0}$ is the initial yield stress at zero plastic strain, $Q_{\infty}$ and $b$ are material parameters. $Q_{\infty}$ is the max. change in the size of the yield surface, and $b$ defines the rate at which the size of the yield surface changes as plastic straining develops. In this paper, the material parameters of LY-100 are listed in Table-1

Table 1. Material properties

\begin{tabular}{ccccccc}
\hline $\begin{array}{c}E \\
(G P a)\end{array}$ & $v$ & $\begin{array}{c}\sigma_{0}\left(\sigma_{0.2}\right) \\
(M P a)\end{array}$ & $\begin{array}{c}C \\
(G P a)\end{array}$ & $\gamma$ & $\begin{array}{c}Q_{\infty} \\
(M P a)\end{array}$ & $b$ \\
\hline 206 & 0.306 & 80.1 & 24.5 & 400 & 200 & 4.5 \\
\hline
\end{tabular}

\subsection{Analysis result and comment}

Figure 4 shows typical hysteretic curve of the shear load versus displacement of the initial shape of SPD compared with corresponding experimental curve. As shown in Figure 4, hysteretic curve obtained from the analysis agree generally well with those from the experiment. The accumulative absorbing energy is shown in Figure 5. In the Figure 5, difference between the experiment and analysis at the last stage is less than $5 \%$. It is clarified that the present analysis with the combined isotropic/kinematic hardening model can accurately predict the cyclic elasto-plastic behavior of the LY100 SPD. In the experimental investigation, as shown in Figure 6, fracture was found at the diagonal corners in the 4th cycle of loading, and progresses with increasing of plastic strain, finally resulted in destruction. Figure 7 shows the accumulated equivalent plastic strain distribution by FEM simulation in the 4th cycle loading, and remarkable strain concentration at the panel corners can be observed.

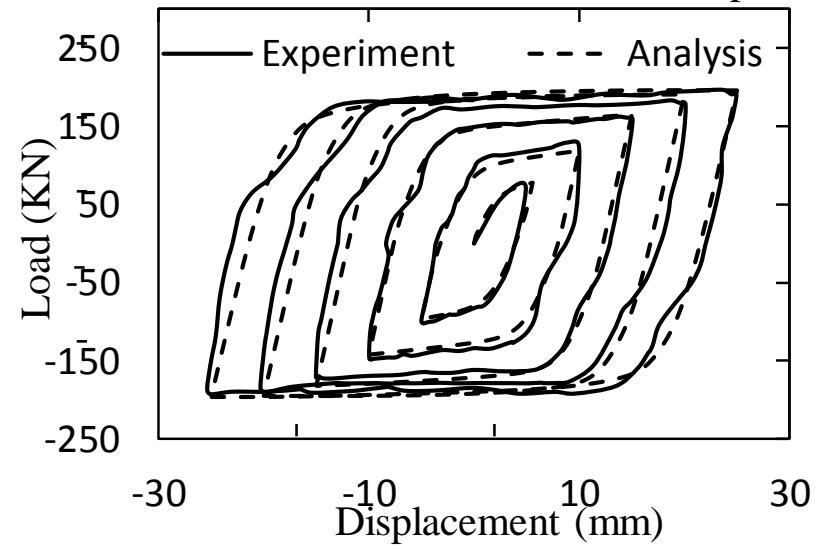

Figure 4. Hysteretic load-displacement curves

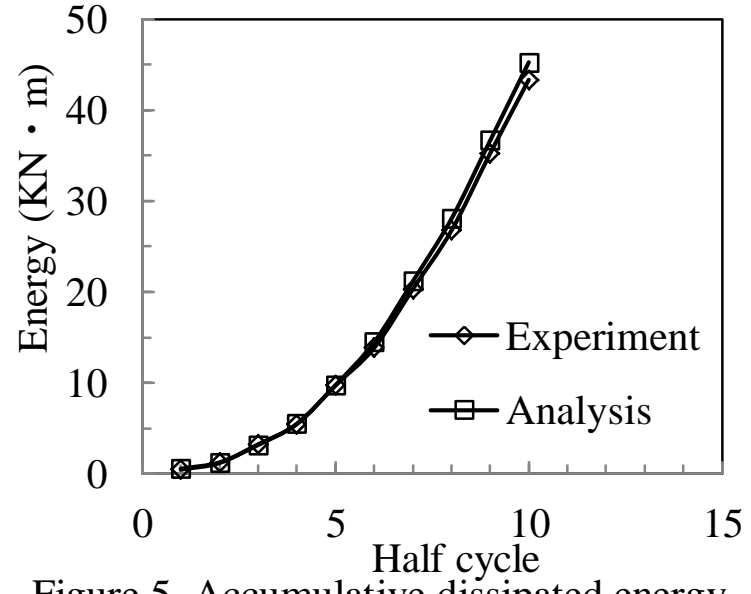

Figure 5. Accumulative dissipated energy 


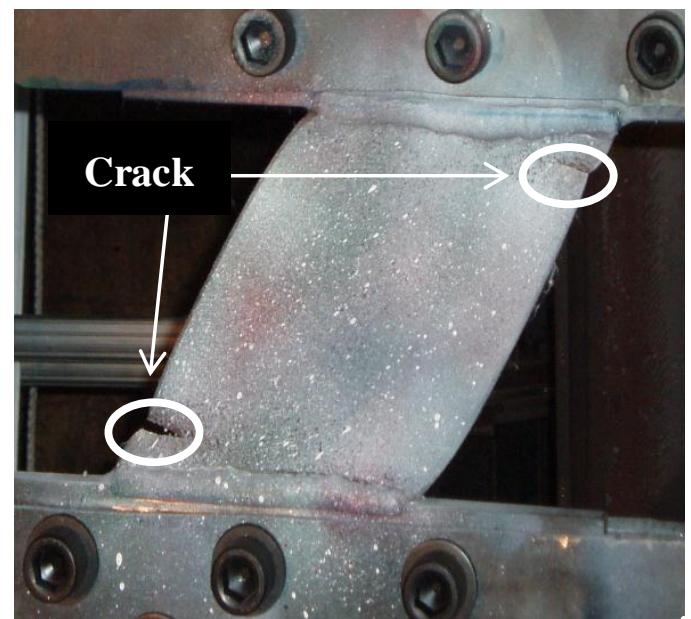

Figure 6. Deformed quadratic SPD

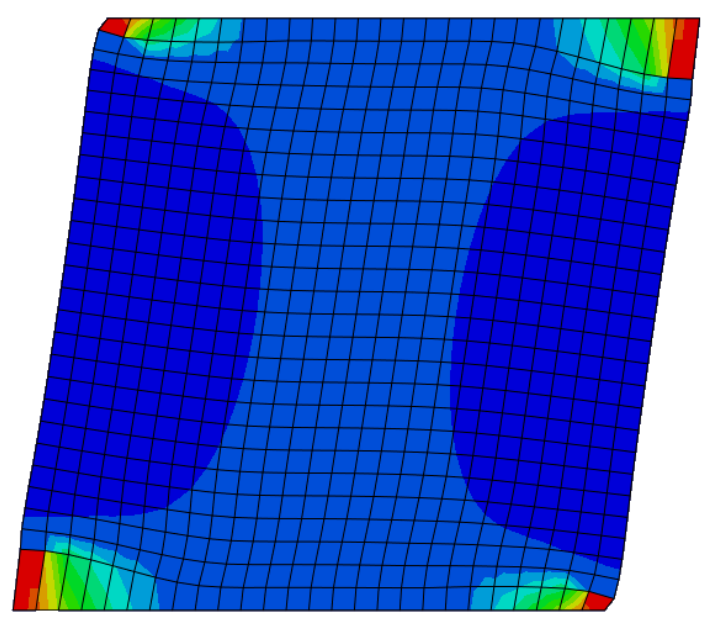

Figure 7. Accumulated equivalent plastic strain distribution at loading point $\mathrm{A}$ in Figure 2

\subsection{Shape parameters}

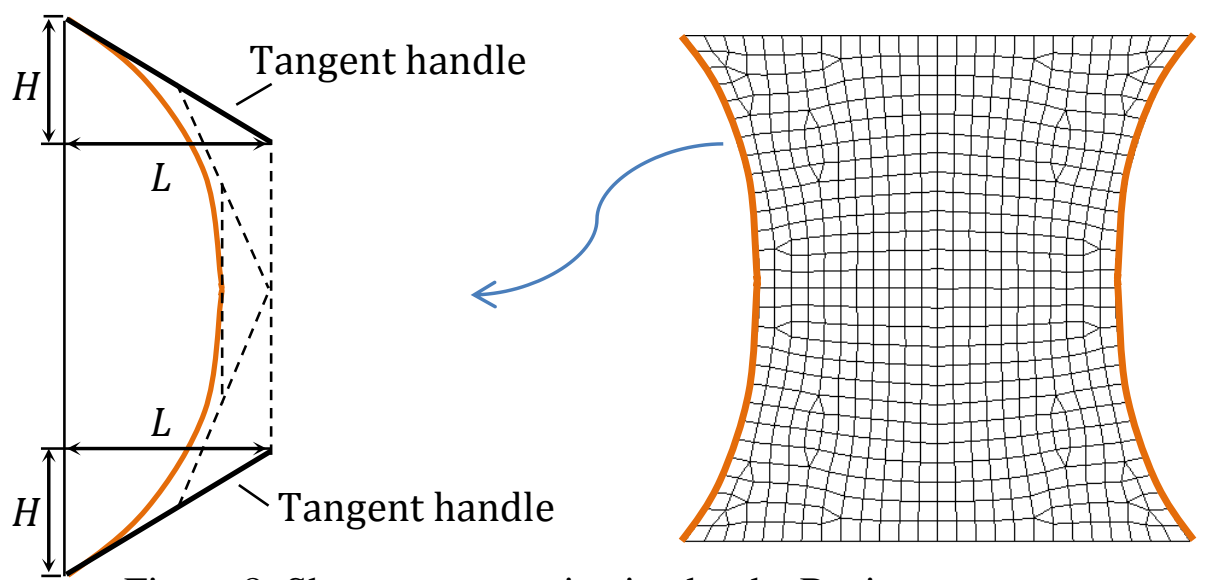

Figure 8 . Shape parameterization by the Bezier curve

As a result in the previous experimental studies by one of the authors, reducing the free edges on the left and right side of the panel can contribute to effectively increase the deformation capacity[1][2] . Therefore, shape of the reduced edge can be described by the Bezier curve and parameterized by two parameters as shown in Figure 8. These parameters $H, L$ indicate projection scales of the tangent handle to the vertical and horizontal directions respectively.

\section{MULTI-OBJECTIVE DESIGN OPTIMIZATION}

In this study, with the aim of the minimizing the max. accumulated plastic equivalent strain and the maximizing the total absorbing energy of SPD, the shape parameters $H, L$ are taken as design variables and an orthogonal array in the design of experiment is employed to assign analysis points in simulating the cyclic elasto-plastic behavior of SPD. Based on the 
numerical results of the cyclic elasto-plastic analysis, the response surface approximation technique is applied to generate the regression equations of the max. accumulated equivalent plastic strain and the absorbing energy in terms of the design variables that are evaluated to be significant at high levels for the response by means of analysis of variance. Then, the regression equations are applied to constitute the multi-objective function by using the weighed sum method as follows:

$$
\begin{gathered}
\text { Maximize } f(L, H)=C_{1} E(H, L) / E_{t}-C_{2} A E S P_{\max } / A E S P_{t} \\
C_{1}+C_{2}=1
\end{gathered}
$$

where $E(H, L), A E S P_{\max }$ indicate the total absorbing energy and max. accumulated equivalent plastic strain at left and right side of SPD, respectively, and $E_{t}, A E S P_{t}$ are preliminary target values. $C_{1}, C_{2}$ indicate the weighting coefficients, which have relationship shown in Equation (4). In this study, the values of $E_{t}, A E S P_{t}, C_{1}, C_{2}$ are taken as $62 \mathrm{KJ}, 200 \%, 0.4$, and 0.6 , respectively.

\subsection{Experimental design}

The experimental design levels of the process variables in the first iteration are shown in Table 2. As shown in Table 2, an orthogonal array $L_{9}$ is employed to arrange the design point, and results of $A E S P_{\max }$ are obtained by the cyclic elasto-plastic analysis at each design point under the same cyclic lateral load shown in Figure 2.

Table 2. Design levels numerical solutions for SPDs

\begin{tabular}{cccccc}
\hline \multirow{2}{*}{ Iteration } & $\begin{array}{c}\text { Design } \\
\text { Point }\end{array}$ & $\begin{array}{c}H \\
(\mathrm{~mm})\end{array}$ & $\begin{array}{c}L \\
(\mathrm{~mm})\end{array}$ & AESP $_{\text {max }}$ & $\begin{array}{c}E \\
(\mathrm{KJ})\end{array}$ \\
\hline \multirow{5}{*}{$1 \mathrm{st}$} & 1 & 20 & 40 & 3.90 & 63.4 \\
& 2 & 20 & 80 & 3.87 & 63.6 \\
& 3 & 20 & 120 & 9.54 & 62.4 \\
& 4 & 30 & 40 & 3.09 & 58.1 \\
& 5 & 30 & 80 & 2.33 & 62.6 \\
& 6 & 30 & 120 & 4.56 & 64.8 \\
& 7 & 40 & 40 & 3.65 & 50.9 \\
& 8 & 40 & 80 & 3.36 & 56.2 \\
\hline
\end{tabular}

\subsection{Analysis of variance (ANOVA)}

The experimental analysis of variance and 3D response surface were carried out to perform the regression analysis of the experimental data and the second-order polynomial model equations were fitted to obtain the regression equations. The fitted polynomial equa- 
tions were then expressed in the form of contour and surface plots in order to illustrate the relationship between the responses and experimental levels of each of variables utilized in this study.

Equation (5) describes the regression model of the present system, which includes the interaction term [8]:

$$
Y=\beta_{0}+\sum_{i=1}^{k} \beta_{i} X_{i}+\sum_{i=1}^{k} \beta_{i i} X_{i}^{2}+\sum \sum_{i<j}^{k} \beta_{i j} X_{i} X_{j}+\varepsilon
$$

where $Y$ is the predicted response $E(H, L)$ or $A E S P_{\max }(H, L)$ in this study and $X_{i}, X_{j}$ are the coded levels of the independent factors $H, L$, respectively. $\beta_{0}, \beta_{i}\left(\beta_{j}\right), \beta_{i j}$ indicate the intercept term, the coefficient for linear effects and the coefficient for interaction effects, respectively. $\varepsilon$ denotes the random error.

Based on the numerical solutions of the cyclic elasto-plastic analysis in the first iteration as shown in Table 2, the response surface regression procedure was employed to fit the polynomial Equation (5) to the numerical analysis results, and the max. accumulated plastic strain $A E S P_{\text {max }}$, the total absorbing energy $E$ are approximated in the form of orthogonal polynomials as:

$$
\begin{array}{r}
A E S P_{\max }(H, L)=-8.248+1.16 H-0.02191 H^{2}+0.522925 L-0.0018825 L^{2} \\
-0.04980062 H L+2.542031 \mathrm{E}-4 H L^{2}+9.335625 \mathrm{E}-4 H^{2} L-5.345312 \mathrm{E}-6 H^{2} L^{2} \\
E(H, L)=93.73507-1.985338 H+1.930768 \mathrm{E}-02 H^{2}-0.7382053 L+0.002365144 L^{2} \\
+0.05766564 H L-2.200206 \mathrm{E}-4 H L^{2}-8.88549 \mathrm{E}-4 H^{2} L+3.94997 \mathrm{E}-6 H^{2} L^{2}
\end{array}
$$

Figure 9 presents the three-dimensional response surface plots for the max. accumulated equivalent plastic strain $A E S P_{\text {max }}(H, L)$ response and the total absorbing energy, $E$ response in terms of the process variables $H, L$ in the first iteration. Figure 9(a) shows that, when the shape parameter $H$ is between $20 \sim 30 \mathrm{~mm}, A E S P_{\max }$ decreases obviously whit increase of $H$ as well as decrease of the shape parameter $L$. On the other hand, it can also be observed that, when $H$ increases to over $30 \mathrm{~mm}$, increasing $L$ can contributes to decrease the $A E S P_{\max }$. It is clear that interaction between the shape parameters $H$ and $L$ is significant and must be considered. Figure 9(b) shows that, the total absorbing energy $E$ decreases with increase of the shape parameter $H$ and decrease of the shape parameter $L$, especially when $H$ increases to over $30 \mathrm{~mm}$. Interaction of $H$ and $L$ is confirmed not significant on $E$.

\subsection{Optimization results and comments}

To obtain more precise approximated response surface, analysis points are selected for the 1 st, 2 nd, 3rd and 4th intration of the optimization process, and the optimum results of the 4 iterations are shown in Table 3. Calculated results of the max. accumulated equivalent plastic strain $A E S P_{\text {max }}$ and the total absorbing energy $E$ at the design points in each optimization iteration are shown in Figure 10. It is observed that the minimization of $\operatorname{AESP}_{\max }(H, L)$ and maximization of $E(H, L)$ are almost obtained simultaneously, and the two evaluation in terms 


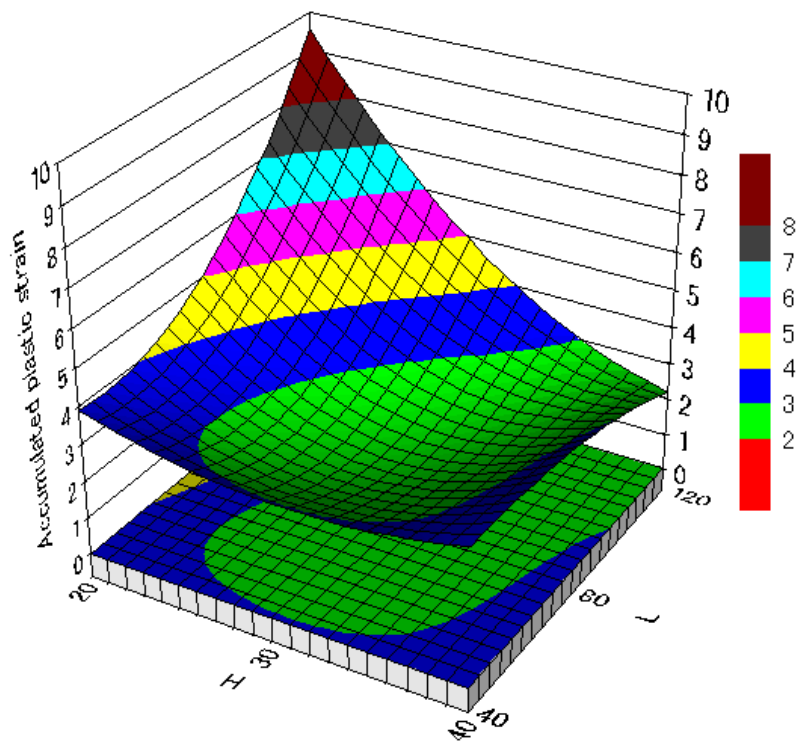

(a) Max. accumulated equivalent plastic strain

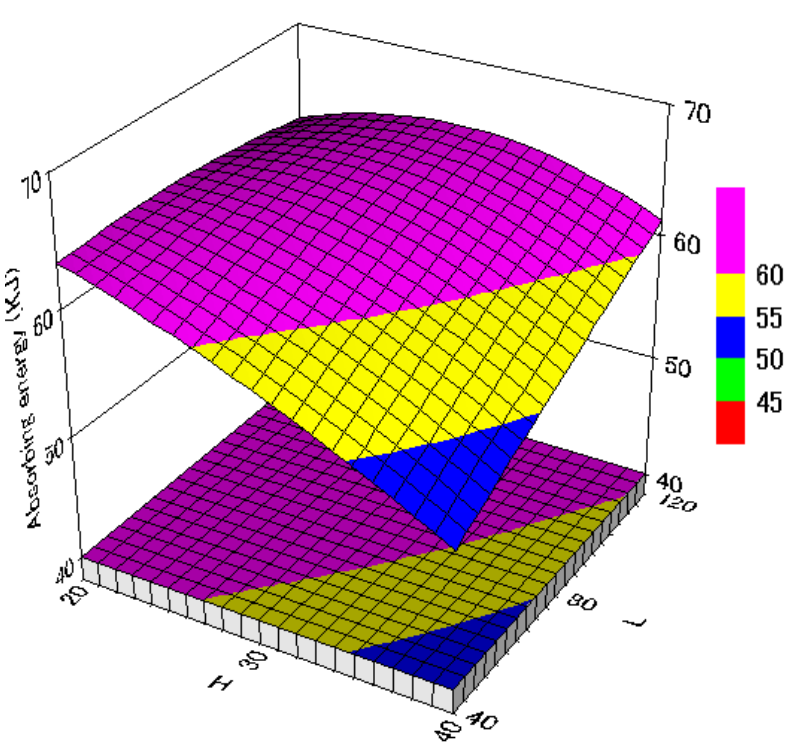

(b) Total absorbing energy

Figure 9. Response surface plot in the 1st iteration

of the shape parameters $H, L$ do not involve a relationship of trade-off generally. Figure 11 shows the iteration convergence histories of the multi-objective function $f(H, L)$.

The obtained optimal shape of SPD is shown in Figure 12. Figure 13 shows accumulated plastic distribution, which is simulation result of the cyclic elasto-plastic behavior of the optimized SPD subjected to cyclic loading. The numerical estimation of max. accumulated equivalent plastic strain at left and right side of the optimized SPD is 1.83 , which is $82.2 \%$ down than value of the SPD with initial shape. It is obvious that the optimal shape can substantially increase the deformation capacity of SPD. Total absorbing energy of the optimized SPD is $63.9 \mathrm{KJ}$, which is $2.3 \%$ lower than the initial one in spite of the volume is about $34 \%$ decrease. It can be considered that energy absorbing behavior of SPD with the optimal shape is more efficient.

Table 3. Optimum results for each iteration (Initial shape: $A E S P_{\max }: 10.28 \quad E: 65.4 K J$ )

\begin{tabular}{|c|c|c|c|c|c|}
\hline & Iteration & $\begin{array}{c}H \\
(\mathrm{~mm})\end{array}$ & $\begin{array}{c}L \\
(\mathrm{~mm})\end{array}$ & $A E S P_{\max }$ & $\begin{array}{c}E \\
(K J)\end{array}$ \\
\hline \multirow{2}{*}{$1 \mathrm{st}$} & Design levels & $20,30,40$ & $40,80,120$ & & \\
\hline & Optimization results & 31.3 & 73 & 2.62 & 61.6 \\
\hline \multirow{2}{*}{ 2nd } & Design levels & $26,31,36$ & $53,73,93$ & & \\
\hline & Optimization results & 33.0 & 93 & 2.46 & 62.9 \\
\hline \multirow{2}{*}{$3 \mathrm{rd}$} & Design levels & $30,33,36$ & $93,103,113$ & & \\
\hline & Optimization results & 34.1 & 113 & 1.89 & 63.6 \\
\hline \multirow{2}{*}{4 th } & Design levels & $31,34,37$ & $113,118,123$ & & \\
\hline & Optimization results & 35.4 & 119 & 1.83 & 63.9 \\
\hline
\end{tabular}




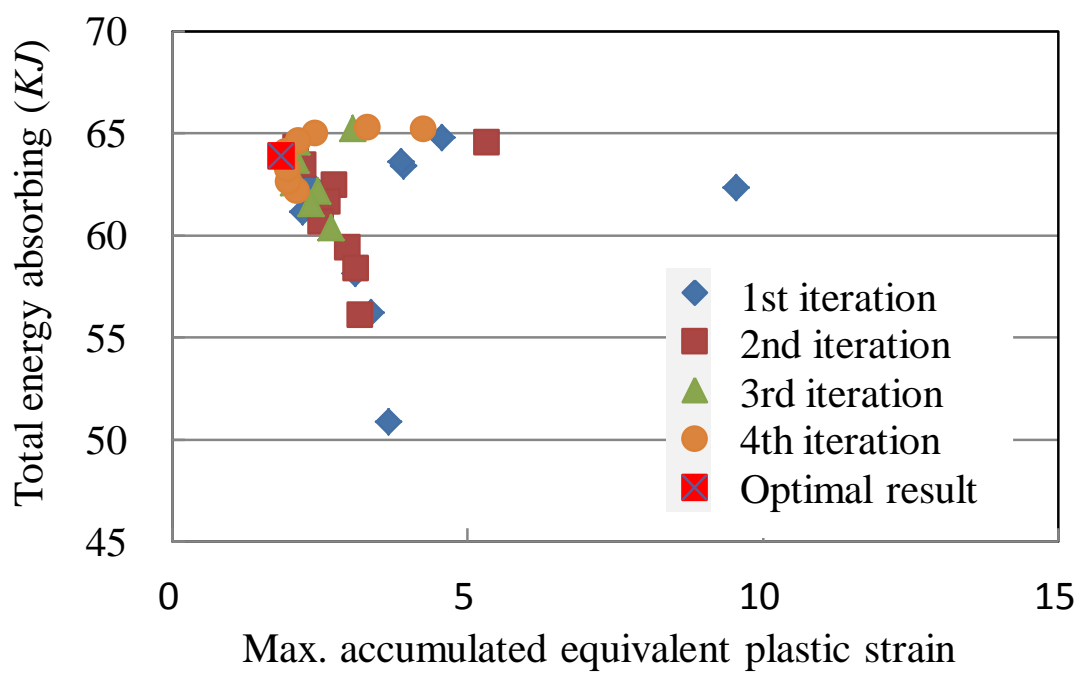

Figure 10. Calculated results of L9 in each iteration

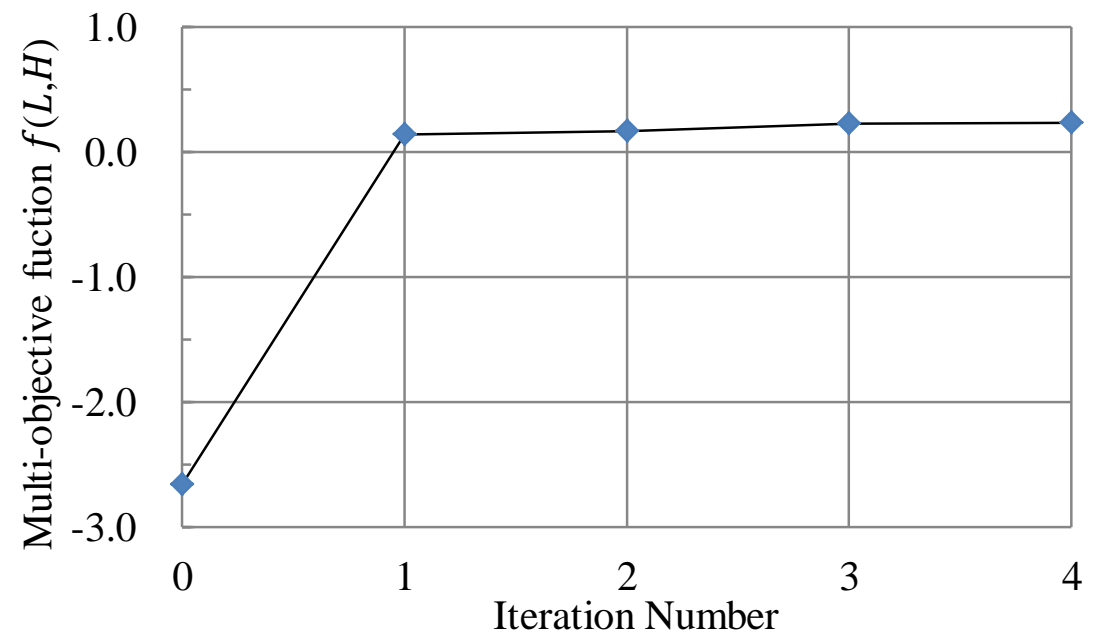

Figure 11. Iteration history of multi-objective optimization problem

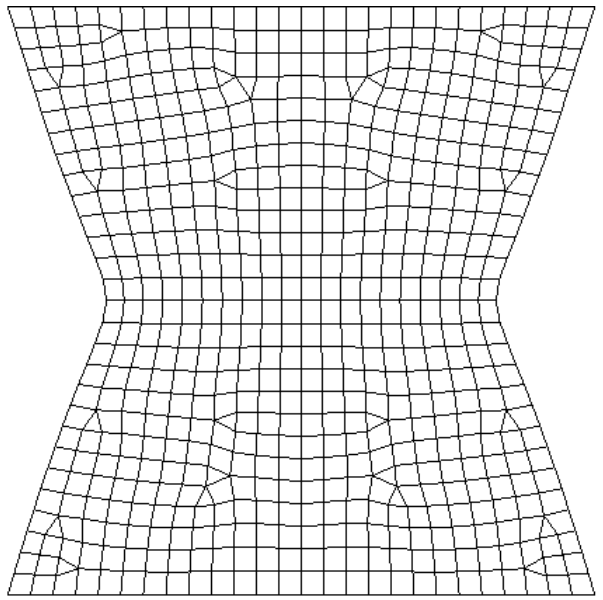

Figure 12. Optimal shape of SPD

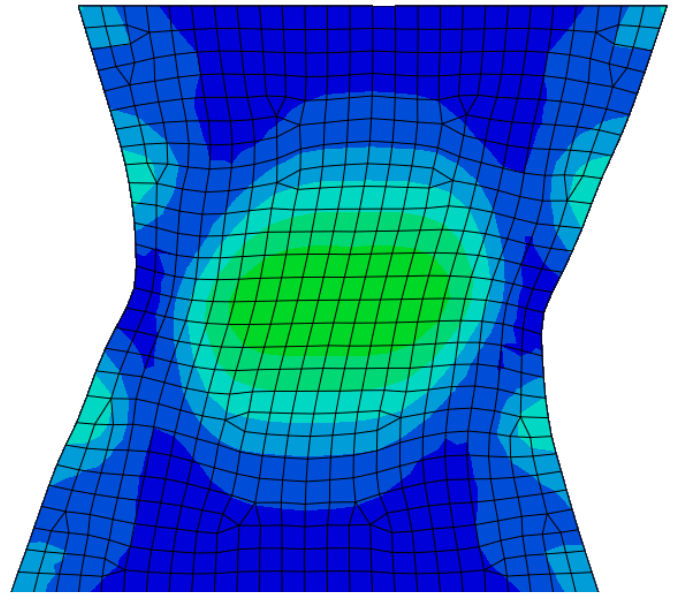

Figure 13. Accumulated equivalent plastic strain distribution of the optimized SPD at loading point B in Figure 2 


\section{CONCLUSIONS}

In this study, a multi-objective design optimization of the low-yield steel SPD, considering the max. accumulated equivalent plastic strain and the total absorbing energy, was carried out to determine the optimal shape parameters. It is confirmed that the optimization methodology with combination between RSM and cyclic elasto-plastic behavior simulation of SPD by FEM is serviceable and effective. As a optimization result, max. accumulated equivalent plastic strain of the optimal shape is $82.2 \%$ decrease that can substantially increase the deformation capacity of SPD, and the maximum of total absorbing energy was almost obtained simultaneously.

\section{Acknowledgements}

This research was supported by grants-in-aid from the Sustainable Mechanical Systems R\&D Center at Toyota Technological Institute.

\section{REFERENCES}

[1] Yang Liu, Tetsuhiko Aoki, Tatsumasa Takaku, Yuhshi Fukumoto, "Cyclic loading tests of shear panel damper made of low yield steel” J. of Structural Eng., JSCE., Vol.53A, No612, pp.560-567, 2007.

[2] T. Aoki, Yang Liu, T. Takaku, and Y. Fukumoto, "Experimental investigation of tapered shear-type seismic devices for bridge bearings". Pacific Structural Steel Conference 2007, Steel Structures in Natural Hazards, Wairakei, New Zealand, Vol.1, 111-117, 2007.

[3] Koike, Y., Yanaka, T., Usami, T., Ge, H.B., Oshita, S., Sagou, D. and Uno, Y., “An Experimental Study on Developing High-Performance Stiffened Shear Panel Dampers", J. of Structural Engineering, JSCE, Vol.54A, pp.372-381, 2008.

[4] Masatoshi Shimoda, Hideyuki Azegami, Toshiaki Sakurai, "Traction Method Approach to Optimal Shape Design Problems”, Journal of Passenger Cars, SAE 1997 Transactions, pp. 2355-2365, 1998.

[5] Hisashi Ihara, Hideyuki Azegami, Masatoshi Shimoda, "Solution to Shape Optimization Problems Considering Material Nonlinearity", Computer Aided Optimization Design of Structures VI, Chap. 3, Sec. 4, pp. 87-96, 1999.

[6] Han J., Yamazaki K., "A study on maximization of dynamic crushing energy absorption of square tubes with and without stiffener". JSME Int, J. Ser. A43 (2), 138-145, 2000.

[7] ABAQUS, Inc., ABAQUS/Analysis user's manual-version 6.5

[8] R. M. Myers, D. C. Montgomery, Response Surface Methodology, John Wiley \& Sons, New York, 2002 